\title{
Apparent Diffusion Coefficient Value Changes and Clinical Correlation in 90 Cases of Cytomegalovirus-Infected Fetuses with Unremarkable Fetal MRI Results
}

\author{
(D)D. Kotovich, DJ.S.B. Guedalia, (D) C. Hoffmann, DG. Sze, (D) A. Eisenkraft, and (D) G. Yaniv
}

\begin{abstract}
BACKGROUND AND PURPOSE: Cytomegalovirus is the leading intrauterine infection. Fetal MR imaging is an accepted tool for fetal brain evaluation, yet it still lacks the ability to accurately predict the extent of the neurodevelopmental impairment, especially in fetal MR imaging scans with unremarkable findings. Our hypothesis was that intrauterine cytomegalovirus infection causes diffusional changes in fetal brains and that those changes may correlate with the severity of neurodevelopmental deficiencies.
\end{abstract}

MATERIALS AND METHODS: A retrospective analysis was performed on 90 fetal MR imaging scans of cytomegalovirus-infected fetuses with unremarkable results and compared with a matched gestational age control group of 68 fetal head MR imaging scans. ADC values were measured and averaged in the frontal, parietal, occipital, and temporal lobes; basal ganglia; thalamus; and pons. For neurocognitive assessment, the Vineland Adaptive Behavior Scales, Second Edition (VABS-II) was used on 58 children in the cytomegalovirus-infected group.

RESULTS: ADC values were reduced for the cytomegalovirus-infected fetuses in most brain areas studied. The VABS-II showed no trend for the major domains or the composite score of the VABS-II for the cytomegalovirus-infected children compared with the healthy population distribution. Some subdomains showed an association between ADC values and VABS-II scores.

CONCLUSIONS: Cytomegalovirus infection causes diffuse reduction in ADC values in the fetal brain even in unremarkable fetal MR imaging scans. Cytomegalovirus-infected children with unremarkable fetal MR imaging scans do not deviate from the healthy population in the VABS-II neurocognitive assessment. ADC values were not correlated with VABS-II scores. However, the lack of clinical findings, as seen in most cytomegalovirus-infected fetuses, does not eliminate the possibility of future neurodevelopmental pathology.

ABBREVIATIONS: $\mathrm{CMV}$ = cytomegalovirus; feMRI = fetal head MR imaging; VABS-II = Vineland Adaptive Behavior Scales, Second Edition

C tomegalovirus (CMV) infection is the most common intrauterine infection, with an overall birth prevalence of $1 \%$ (range, $0.2 \%-2.5 \%) .{ }^{1,2}$ Only $10 \%-15 \%$ of the infected fetuses are symptomatic at birth, presenting with typical clinical findings of congenital infection, ${ }^{3,4}$ while an additional 10\%-15\% of infants develop the symptoms during the first years of life. ${ }^{1,2,5,6}$ The

Received December 14, 2016; accepted after revision March 6, 2017.

From The Faculty of Medicine (D.K., A.E.), Institute for Research in Military Medicine, The Hebrew University of Jerusalem, Jerusalem, Israel; The Israel Defense Forces Medical Corps (D.K., A.E.), Chevy Chase, Maryland; Neuropsychology Unit (I.S.B.G., G.Y.), Shaare Zedek Medical Center, Jerusalem, Israel; Department of Diagnostic Imaging (C.H.) and Dr. Pinchas Bornstein Talpiot Medical Leadership Program (G.Y.), Sheba Medical Center, Tel HaShomer, Ramat Gan, Israel; and Department of Radiology and Biomedical Imaging (G.S., G.Y.), Yale School of Medicine, New Haven, Connecticut.

Please address correspondence to Gal Yaniv, MD, PhD, 154 Willard St, New Haven, CT 06515; e-mail: Gal.yaniv@yale.edu

Indicates article with supplemental on-line tables.

Indicates article with supplemental on-line photos.

http://dx.doi.org/10.3174/ajnr.A5222 clinical findings include, but are not limited to, intrauterine growth restriction, periventricular calcifications, microcephaly, ventriculomegaly, hepatosplenomegaly, and cardiovascular system anomalies. ${ }^{3,4,7}$

Most symptomatic infants will have long-term sequelae, including neurodevelopmental damage with intellectual disabilities, ranging up to severe decreases in cognitive capacity. ${ }^{2,4,5,8,9}$ Asymptomatic neonates constitute most cases, up to $90 \%$ of the infected fetuses, with outcomes still unclear due to limited research. ${ }^{1,5,6,8,9}$

Sonography is a widely used prenatal screening tool and can show typical findings suggestive of CMV infection. Recent studies have shown that sonography is not sensitive enough for the entire spectrum of neuropathologies, mainly brain maturation. ${ }^{10,11}$ Fetal head MR imaging (feMRI) is accepted as a complementary test for the evaluation of the brain. Studies have shown that feMRI produces much more information, including improved spatial resolution, visualization of the entire brain parenchyma, and detection of white matter maturation and pathologies earlier and better than sonogra- 

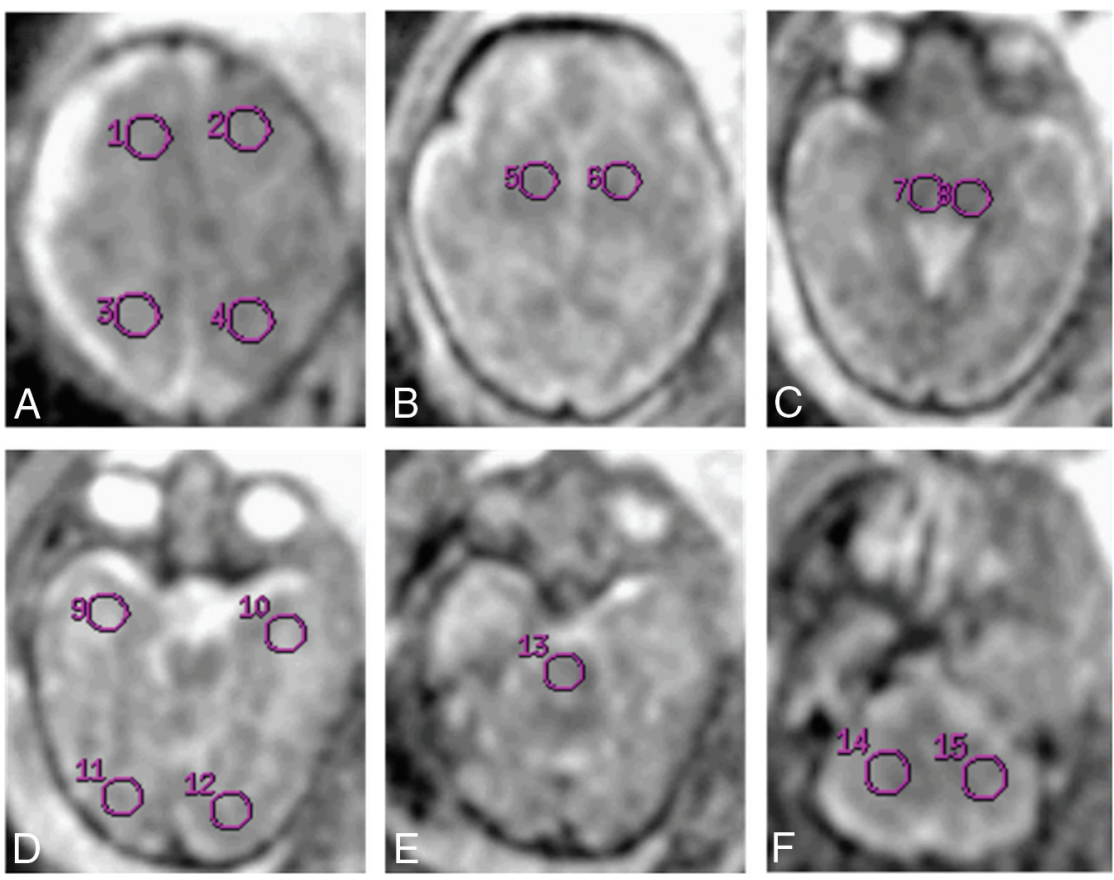

FIG 1. ROI analyzed for apparent diffusion coefficient. ROI placement example. $A, 1,2=$ frontal lobe; $3,4=$ parietal lobe. $B, 5,6=$ basal ganglia. $C, 7,8=$ thalami. $D, 9,10=$ temporal lobe; $11,12=$ occipital lobe. $E, 13=$ pons. $F, 14,15=$ cerebellum.

phy. ${ }^{10-13}$ However, even with both techniques combined, it is still unclear how to accurately predict the extent of the neurodevelopmental impairment in the prenatal period, especially in cases without any notable imaging pathology..$^{10,11,13,14}$

Diffusion-weighted imaging (DWI, DTI) was studied extensively for its utility in the evaluation of the normal development of the fetal brain. ${ }^{15-20}$ One of the DWI metrics, the apparent diffusion coefficient, allows quantitative evaluation of cerebral maturation and intracellular changes in utero. ${ }^{15,18-22}$ Very little research has been done examining the ADC values in CMV-infected fetal brains, and even less research has focused on fetuses with normal feMRI results. ${ }^{14,18}$

In our current study, we compared ADC values in several anatomic brain areas of CMV-infected fetuses with unremarkable feMRI results and an age-matched control group with normal MR imaging findings. For neurodevelopmental assessment, the Vineland Adaptive Behavior Scales, Second Edition (VABS-II) ${ }^{23,24}$ was performed on children from the CMV-infected group.

Our hypothesis was that CMV-infection causes diffusional changes in fetal brains and that those changes may be correlated to the severity of neurodevelopmental deficiencies.

\section{MATERIALS AND METHODS}

This retrospective study was approved by the institutional review board of Tel HaSomer Hospital.

\section{Subjects and Procedures}

A retrospective analysis was performed on 90 sequential feMRI scans of 83 polymerase chain reaction-validated CMV-infected fetuses obtained between 2004 and 2014. Five fetuses had 2 scans, and one fetus had 3 scans. Any fetus with a major abnormality on the feMRI scan was excluded from this study.
The control group included $68 \mathrm{fe}$ tuses scanned between 2006 and 2013 who had unremarkable feMRI results, with no clinical or laboratory evidence of chromosomal abnormalities or intrauterine infection. The indications for the feMRI scan were the following: a sibling with perinatal neurologic findings, previous abnormal pregnancy, prenatal sonography with abnormal findings not confirmed by feMRI, or a suspected CMV infection without confirmation by polymerase chain reaction testing of the amniotic fluid.

\section{MR Imaging}

Technical parameters and protocol of the scan are identical to those of our previous published work. ${ }^{25}$ ROIs were placed as shown in Fig 1. No significant difference between the ADC values of the 2 sides (except for the basal ganglia and the occipital lobe in the CMV group and the temporal lobe in the control group) was shown.

\section{Vineland Adaptive Behavior Scales, Second Edition}

The VABS-II test was performed on 58 children from the CMVinfected group whose parents agreed to participate in the study and whom we were able to contact. The questionnaire was performed by telephone with the mother of the child in all the cases, except one with the father alone.

\section{Statistical Methods}

To compare ADC values of the CMV and control groups, we applied the $t$ test. The $\chi^{2}$ test was used for the comparison of the observed and expected frequency of each VABS-II adaptive level. The comparison of ADC values with VABS-II adaptive levels was performed by either applying the ANOVA model with the Scheffe procedure for post hoc comparisons or the Kruskal-Wallis nonparametric test. Nonparametric tests were used when the groups compared were small and the distribution of the variables was not normal.

All tests were 2 -tailed, and a $P$ value of $\leq 5 \%$ was considered statistically significant. The statistical analysis was performed with SPSS, Version 21 (IBM, Armonk, New York).

\section{RESULTS}

\section{Group Characteristics}

The CMV-infected fetuses group consisted of 90 feMRI scans (Table 1) with a mean maternal age of $31.3 \pm 4.2$ years, while the control group consisted of 68 fetal scans with a mean maternal age of $32.2 \pm 4.8$ years $(P>.05)$.

The mean ROI size was $83.5 \pm 4.3$ for the control group and $82.6 \pm 3.34$ for the CMV-infected group $(P>.05)$.

All the CMV-infected fetuses had unremarkable feMRI reports (On-line Table 1).

The Vineland Adaptive Behavior Scales test was performed on 58 
children ( 32 males and 26 females) of the 90 patients in the CMVinfected group, with a mean age of $60.1 \pm 30.8$ months. Eight of the children had hearing disabilities (14\%) (Table 1 and On-line Table 1).

\section{Effect of CMV Infection on Regional ADC}

CMV infection was accompanied by a highly significant decrease in ADC in all brain regions except in the temporal lobes (Table 2 and On-line Table 2). ADC values were significantly reduced in the frontal $(P=.005)$, parietal $(P=.008)$, and occipital $(P=.022)$ lobes; basal ganglia $(P=.016)$; thalamus $(P<.001)$; cerebellum $(P=.005)$; and pons $(P=.011)$. The temporal lobes had no significant decrease, yet they showed a high correlation trend $(P=$ .057) (Fig 2 and On-line Table 2).

\section{VABS-II Results}

The VABS-II scores are summarized in On-line Table 3 for all the domains, subdomains, and the Adaptive Behavior Composite.

Table 1: Descriptive data of the fetuses included in the study

\begin{tabular}{lcc}
\hline & Controls & CMV Group \\
\hline No. (feMRI) & 68 & 90 \\
Maternal age (mean) (yr) & $32.05 \pm 4.43$ & $31.58 \pm 4.23$ \\
GA (MRI) (mean) & $31.8 \pm 2.3$ & $33.1 \pm 2.0$ \\
GA (infection, No.) & NA & \\
1st trimester & & 25 \\
2nd trimester & & 43 \\
3rd trimester & & 15 \\
Unknown & NA & 7 \\
VABS-II & & 58 \\
Child's age (mean) (mo) & & $60.12 \pm 30.774$ \\
Male (No.) & & 32 \\
$\quad$ Mean age (mo) & & $50.75 \pm 26.3$ \\
Female (No.) & 26 \\
$\quad$ Mean age (mo) & & $71.65 \pm 32.4$ \\
Hearing disabilities (No.) & & $8(14 \%)$ \\
\hline
\end{tabular}

Note:-GA indicates gestational age; NA, not applicable.

Table 2: Regional effects of cytomegalovirus infection on fetal brain ADC

\begin{tabular}{lccc}
\hline \multicolumn{1}{c}{ Region } & Controls \pm SD & CMV Group \pm SD & P Value \\
\hline Frontal lobe & $1762.3 \pm 191.6$ & $1675.0 \pm 190.3$ & .005 \\
Parietal lobe & $1733.9 \pm 228.9$ & $1637.2 \pm 220.5$ & .008 \\
Occipital lobe & $1700.2 \pm 193.7$ & $1629.9 \pm 186.7$ & .022 \\
Temporal lobe & $1687.5 \pm 172.5$ & $1636.7 \pm 159.1$ & .057 \\
Basal ganglia & $1448.5 \pm 212.3$ & $1375.2 \pm 147.3$ & .016 \\
Thalamus & $1354.2 \pm 220.7$ & $1247.2 \pm 155.3$ & $<.001$ \\
Cerebellum & $1486.3 \pm 198.7$ & $1407.5 \pm 152.5$ & .005 \\
Pons & $1359.2 \pm 189.3$ & $1286.3 \pm 164.7$ & .011 \\
\hline
\end{tabular}

${ }^{a}$ Data are means. ADC units are $10^{6} \mathrm{~mm}^{2} / \mathrm{s}$

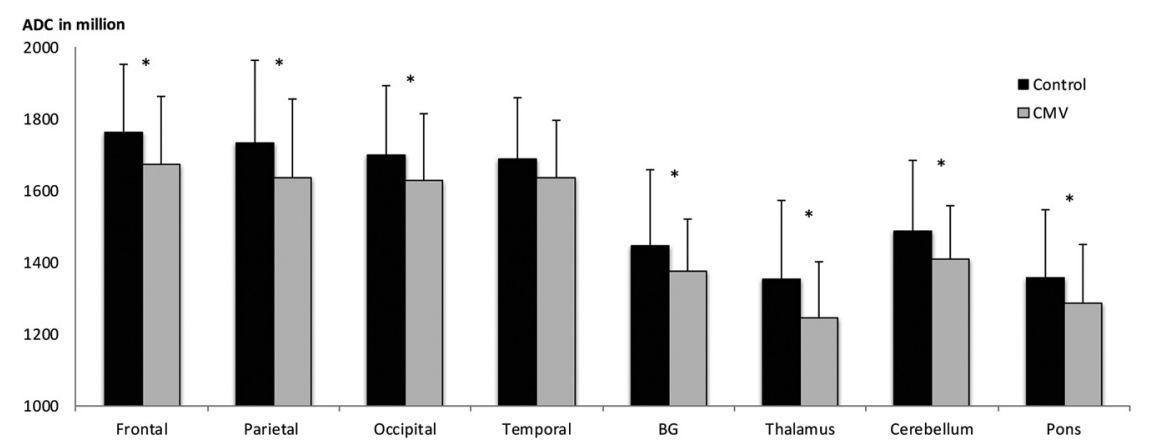

FIG 2. Comparison between ADC values (in millions) of different brain regions from CMV-infected fetuses (gray) and a control group (black). A significant difference is shown with an asterisk.
Significant differences were observed in the following subdomains between the standard score and the CMV-infected results distribution (Fig 3): Written $(P=.001)$ and Coping Skills $(P<.001)$ with a tendency toward lower scores, and in the Receptive subdomain $(P<.001)$ with a tendency toward higher scores. Significant differences were observed in the Daily Living Skills $(P=.011)$ and Socialization $(P=.009)$ domains with the scores accumulating in the adequate category, thus creating a statistically significant difference from the expected normal distribution. The Adaptive Behavior Composite also showed a significant difference $(P=.005)$, with the results again accumulating in the adequate category. The Interpersonal Relationships subdomain was not significantly different, yet it showed a high tendency $(P=$ .054), with a small tendency toward lower scores.

The categories with significance did not change when the statistical analysis was performed with only the 50 children without hearing disabilities.

\section{Correlation between ADC Values and VABS-II: 50 Children (No Hearing Disabilities)}

Correlation between the ADC values and the VABS-II showed a significant difference for the following: pons and Written subdomain $(P=.052)$ with a general negative trend; pons and Daily Living Skills subdomain $(P=.013)$ with a positive trend; basal ganglia and Coping Skills subdomain $(P=.049)$ with no clear trend; and a tendency in the temporal lobe $(P=.065)$ with a general positive trend; temporal lobe and Socialization domain $(P=.043)$ with a tendency in the thalamus $(P=.076)$; frontal/parietal/temporal lobes and the pons with Fine Motor Skills subdomain $(P=.009, P=.05$, and $P=.005$ and $P=$ .048 , respectively) with no clear trend and a tendency in the thalamus and occipital lobe $(P=.06$ and $P=.074$, respectively) with no clear trend; and the pons and Motor Skills domain $(P=.037)$ with no clear trend. A tendency had been shown between the thalamus and the Play and Leisure Time subdomain $(P=.06)$ with a general positive trend and the parietal lobe with Gross Motor subdomain $(P=.064)$ with a general positive trend (Fig 4).

\section{DISCUSSION}

Because the leading intrauterine infection has no effective vaccination in sight and with up to $20 \%-25 \%$ of infected fetuses eventually with some degree of damage, ${ }^{1-6,8,26,27}$ prenatal CMV infection remains a major concern.

To determine the pathology and predict future sequelae, sonography and feMRI are used complementarily. However, the lack of findings, as seen in most CMV-infected fetuses, does not eliminate the possibility of future neurodevelopmental pathology. ${ }^{7,10-13,17}$ This possibility raises the need for more reliable and objective imaging tools for neonatal evaluation to aid clinicians and parents in making more accurate and evidence-based decisions.

ADC measurements could be the tool in this evaluation. As a quantitative value 

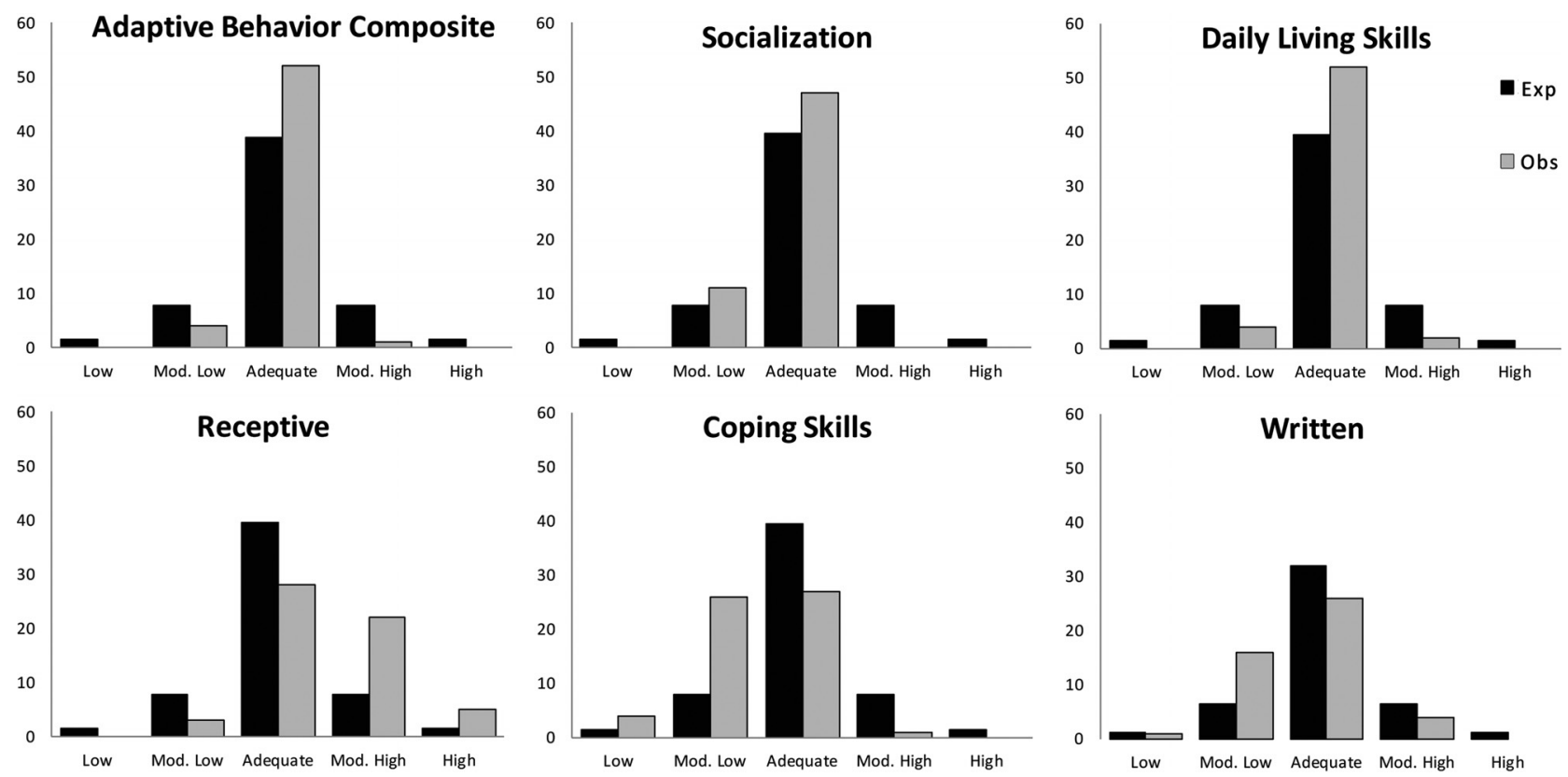

FIG 3. Vineland domains and subdomain absolute results distribution by category of the CMV-infected group (gray) compared with the normal expected distribution (black). Only significant results are shown.
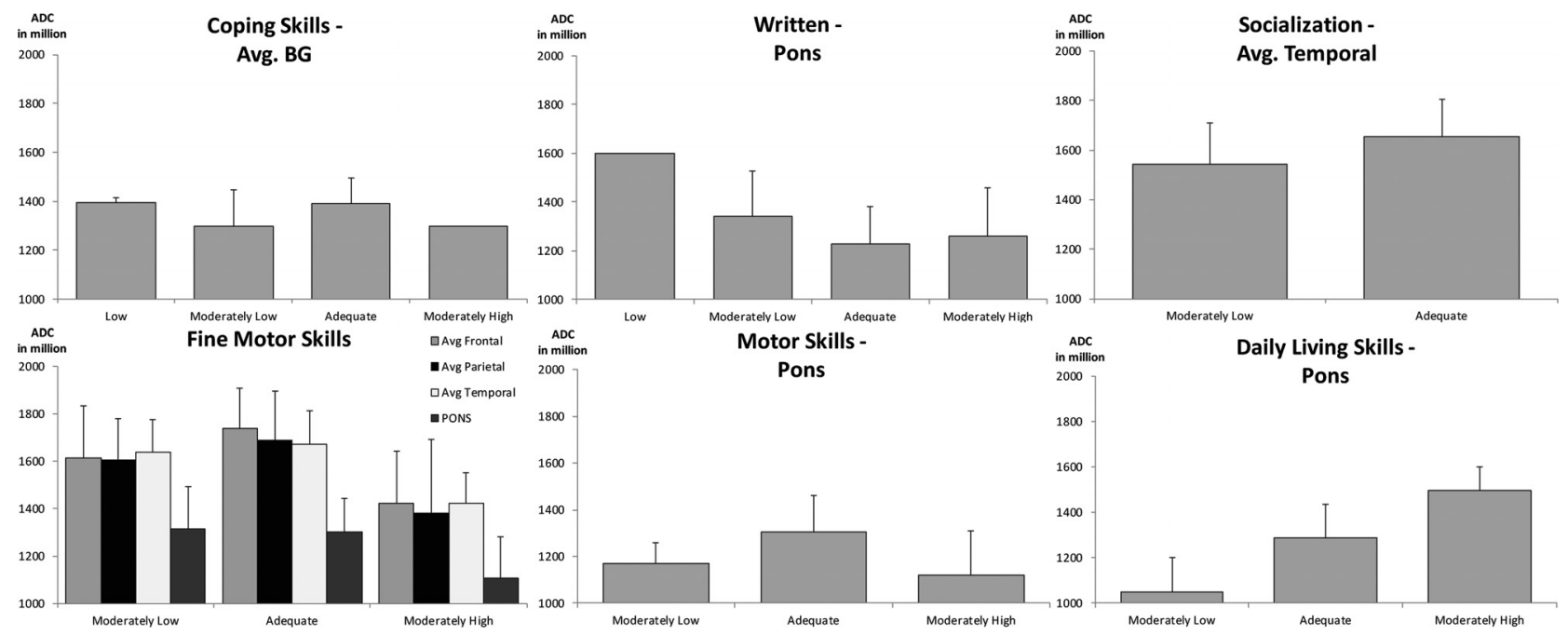

FIG 4. Correlation between ADC values (in millions) by brain regions and Vineland results by domains or subdomains of neonatal CMV-infected children, including only children without hearing disabilities and Vineland results. Only significant results are shown. BG indicates basal ganglia; Avg., average.

measuring voxel diffusion changes, it allows identification of tissue changes at an early stage and quantitative follow-up. ${ }^{14,16-19,22}$

To date, only 3 studies ${ }^{14,28,29}$ have been performed evaluating $\mathrm{ADC}$ values in congenital CMV-infected brains, with 2 of them ${ }^{28,29}$ having a sample size of only 4 and 5 neonates, respectively. The third study ${ }^{14}$ was performed by our department, and part of its sample was included in this study (51/90). However, this is the first time, to our knowledge, that ADC studies of the CMV-infected fetal brains have focused on feMRI scans with unremarkable findings.

We compared the ADC values of confirmed CMV-infected fetal brain regions with unremarkable feMRI findings with those of a matched healthy control group and found significantly diffuse decreased ADC values throughout the fetal brain in the CMV-infected group, except for the temporal lobes $(P=.057)$.
These findings are similar to those in previous studies, ${ }^{14,28}$ though we emphasize that the difference exists even if the feMRI findings are unremarkable.

Analysis of the ADC values shows a significant difference among trimesters in most of the areas examined, excluding the basal ganglia and the pons (On-line Fig 1). Those findings are thought to correspond with previous studies showing a decrease in ADC with fetal brain maturation, mostly due to the ongoing process of myelination. However, this decrease may also reflect microgliosis, which is the hallmark of neuroinflammation of all causes. ${ }^{14,15,18-}$ 22,30,31 These results are shown in unremarkable feMRI findings of CMV-infected fetuses and may imply an underlying inflammatory process within the fetal brain, unrecognized by conventional imaging. 
To assess neurocognitive development in the CMV-infected group, we used the VABS-II, the most commonly used tool for adaptive function assessment, ${ }^{23,24,32}$ and compared the results with the test standardized distribution. The results showed no tendency toward lower or higher scores in the major domains or the composite score. Analysis of the subdomains showed a tendency toward lower scores in the Written and Coping Skills subdomain and a tendency for higher scores in the Receptive subdomain. These results align with some previous studies showing no major difference between neurodevelopmental outcomes of asymptomatic children with CMV and the healthy population, ,9,33 yet they raise the possibility of a deficit as shown in the Written and Coping skills subdomains. A possible explanation for those findings may be higher scores in the Receptive subdomain, which in turn cause the lower scores in the Coping skills subdomain because the child has problems integrating the overflow of information he or she perceives. Future studies focusing on these categories should be performed for further evaluation.

Comparison of the children from the CMV-infected group treated at birth with ganciclovir with the other children showed a benefit for the treatment in the Gross Motor subdomain, with the treated children scoring slightly higher within the normal distribution (On-line Fig 2), thus supporting the current policy of treatment with ganciclovir of asymptomatic neonates. Comparison of the children with hearing disabilities between the CMVinfected group and the other children showed no marked trend, though the small size of the hearing disability group should be borne in mind.

ADC values were evaluated as a potential prognostic factor by matching them with the corresponding Vineland scores. Significant correlation with a coherent trend has been shown for the Daily Living Skills domain and the pons and for the Community domain and the frontal lobe, with lower ADC values matching lower Vineland scores. The remainder of the significant correlation scores of ADC and Vineland have shown no coherent trend.

This work has a few limitations. First, although the CMV and control ADC values statistically differ, the SDs overlap; therefore, no absolute cutoff to distinguish the groups can be used. Nevertheless, single patient follow-up might be useful with further research. The second is the lack of an intellectual function assessment for the CMV-infected children. Intellectual functioning is an essential part of intellectual disability assessment combined with the adaptive behavior, which was assessed with the Vineland test. The third limitation is the number of CMV-infected children with Vineland scores. Due to most children falling into the adequate category, the number of children in the other categories was low; therefore, this number limits the statistical analysis of the results for correlation to ADC values.

\section{CONCLUSIONS}

This is the largest work to date to evaluate the influence of congenital CMV infection on ADC values in the brain on feMRI scans with unremarkable findings and the first to search for a correlation between those values and the cognitive outcome in children. We observed reduced ADC values in most brain areas studied. Neurodevelopmental assessment of the CMV-infected children with unremarkable feMRI findings showed no trend for the major domains or the composite score, with a trend toward lower results in the Coping skills and Written subdomains and a trend toward higher results in the Receptive subdomain. A benefit for treating asymptomatic neonates with ganciclovir was observed for Gross Motor functions.

\section{REFERENCES}

1. Elliott SP. Congenital cytomegalovirus infection: an overview. Infect Disord Drug Targets 2011;11:432-36 Medline

2. Naing ZW, Scott GM, Shand A, et al. Congenital cytomegalovirus infection in pregnancy: a review of prevalence, clinical features, diagnosis and prevention. Aust N Z Obs Gynaecol 2016;56:9-18 CrossRef Medline

3. Ornoy A, Diav-Citrin O. Fetal effects of primary and secondary cytomegalovirus infection in pregnancy. Reprod Toxicol 2006;21:399409 CrossRef Medline

4. Dreher AM, Arora N, Fowler KB, et al. Spectrum of disease and outcome in children with symptomatic congenital cytomegalovirus infection. J Pediatr 2014;164:855-59 CrossRef Medline

5. Zhang XW, Li F, Yu XW, et al. Physical and intellectual development in children with asymptomatic congenital cytomegalovirus infection: a longitudinal cohort study in Qinba mountain area, China. J Clin Virol 2007;40:180-85 CrossRef Medline

6. Goderis J, De Leenheer E, Smets K, et al. Hearing loss and congenital CMV infection: a systematic review. Pediatrics 2014;134:972-82 CrossRef Medline

7. Fink KR, Thapa MM, Ishak GE, et al. Neuroimaging of pediatric central nervous system cytomegalovirus infection. Radiographics 2010;30:1779-96 CrossRef Medline

8. Fowler KB, Boppana SB. Congenital cytomegalovirus (CMV) infection and hearing deficit. J Clin Virol 2006;35:226-31 CrossRef Medline

9. Temple RO, Pass RF, Boll TJ. Neuropsychological functioning in patients with asymptomatic congenital cytomegalovirus infection. J Dev Behav Pediatr 2000;21:417-22 Medline

10. Benoist G, Salomon LJ, Mohlo M, et al. Cytomegalovirus-related fetal brain lesions: comparison between targeted ultrasound examination and magnetic resonance imaging. Ultrasound Obs Gynecol 2008;32:900-05 CrossRef Medline

11. Picone O, Simon I, Benachi A, et al. Comparison between ultrasound and magnetic resonance imaging in assessment of fetal cytomegalovirus infection. Prenat Diagn 2008;28:753-58 CrossRef Medline

12. Lipitz S, Hoffmann C, Feldman B, et al. Value of prenatal ultrasound and magnetic resonance imaging in assessment of congenital primary cytomegalovirus infection. Ultrasound Obs Gynecol 2010;36: 709-17 CrossRef Medline

13. Capretti MG, Lanari M, Tani G, et al. Role of cerebral ultrasound and magnetic resonance imaging in newborns with congenital cytomegalovirus infection. Brain Dev 2014;36:203-11 CrossRef Medline

14. Yaniv G, Hoffmann C, Weisz B, et al. Region-specific reductions in brain apparent diffusion coefficient in cytomegalovirus-infected fetuses. Ultrasound Obs Gynecol 2016;47:600-67 CrossRef Medline

15. Schneider JF, Confort-Gouny S, Le Fur Y, et al. Diffusion-weighted imaging in normal fetal brain maturation. Eur Radiol 2007;17: 2422-29 CrossRef Medline

16. Bydder GM, Rutherford MA. Diffusion-weighted imaging of the brain in neonates and infants. Magn Reson Imaging Clin N Am 2001; 9:83-98, viii Medline

17. Abdelhalim AN, Alberico RA. Pediatric neuroimaging. Neurol Clin 2009;27:285-301, x CrossRef Medline

18. Huppi PS, Inder TE. Magnetic resonance techniques in the evaluation of the perinatal brain: recent advances and future directions. Semin Neonatol 2001;6:195-210 CrossRef Medline

19. Schneider MM, Berman JI, Baumer FM, et al. Normative apparent diffusion coefficient values in the developing fetal brain. AJNR AmJ Neuroradiol 2009;30:1799-803 CrossRef Medline 
20. Gropman A. Imaging of neurogenetic and neurometabolic disorders of childhood. Curr Neurol Neurosci Rep 2004;4:139-46 Medline

21. Counsell SJ, Allsop JM, Harrison MC, et al. Diffusion-weighted imaging of the brain in preterm infants with focal and diffuse white matter abnormality. Pediatrics 2003;112(1 pt 1):1-7 Medline

22. Miller SP, Vigneron DB, Henry RG, et al. Serial quantitative diffusion tensor MRI of the premature brain: development in newborns with and without injury. J Magn Reson Imaging 2002;16:621-32 CrossRef Medline

23. Sparrow SS, Cicchetti DV. Diagnostic uses of the Vineland Adaptive Behavior Scales. J Pediatr Psychol 1985;10:215-25 CrossRef Medline

24. Scattone D, Raggio DJ, May W. Comparison of the Vineland Adaptive Behavior Scales, Second Edition, and the Bayley Scales of Infant and Toddler Development, Third Edition. Psychol Rep 2011;109: 626-34 CrossRef Medline

25. Yaniv G, Katorza E, Bercovitz R, et al. Region-specific changes in brain diffusivity in fetal isolated mild ventriculomegaly. Eur Radiol 2016;26:840-48 CrossRef Medline

26. Kenneson A, Cannon MJ. Review and meta-analysis of the epidemiology of congenital cytomegalovirus (CMV) infection. Rev Med Virol 2007;17:253-76 CrossRef Medline

27. Townsend CL, Forsgren M, Ahlfors K, et al. Long-term outcomes of congenital cytomegalovirus infection in Sweden and the United Kingdom. Clin Infect Dis 2013;56:1232-39 CrossRef Medline

28. Hoffmann C, Weisz B, Lipitz S, et al. Regional apparent diffusion coefficient values in 3rd trimester fetal brain. Neuroradiology 2014; 56:561-67 CrossRef Medline

29. van der Voorn JP, Pouwels PJ, Vermeulen RJ, et al. Quantitative MR imaging and spectroscopy in congenital cytomegalovirus infection and periventricular leukomalacia suggests a comparable neuropathological substrate of the cerebral white matter lesions. Neuropediatrics 2009;40:168-73 CrossRef

30. Hüppi PS, Dubois J. Diffusion tensor imaging of brain development. Semin Fetal Neonatal Med 2006;11:489-97 Medline

31. Lodygensky GA, West T, Moravec MD, et al. Diffusion characteristics associated with neuronal injury and glial activation following hypoxia-ischemia in the immature brain. Magn Reson Med 2011;66: 839-45 CrossRef Medline

32. Ray-Subramanian CE, Huai N, Ellis Weismer S. Brief report: adaptive behavior and cognitive skills for toddlers on the autism spectrum. J Autism Dev Disord 2011;41:679-84 CrossRef Medline

33. Farkas N, Hoffmann C, Ben-Sira L, et al. Does normal fetal brain ultrasound predict normal neurodevelopmental outcome in congenital cytomegalovirus infection? Prenat Diagn 2011;31:360-66 CrossRef Medline 